\title{
Thermal Conductivity of Cross-linked Polymers
}

\author{
Osamu Yамамото \\ Polymer Research Division, Department of Materials, \\ Institute of Space and Aeronautical Science, \\ University of Tokyo, Komaba, Meguro-ku, \\ Tokyo, Japan.
}

(Received February 22, 1971)

\begin{abstract}
Thermal conductivity of cross-linked polymers was studied theoretically. A theory derived from the network model, which consists of thermal conduction through van der Waals and primary bonds, predicts that the thermal conductivity increases with an increasing degree of cross-linking. Calculations for estimating the effect of crosslinking on the thermal conductivity were made for polystyrene and poly(methyl methacrylate). Thermal conduction through the van der Waals and primary bonds was also discussed using the Debye formula for the thermal conductivity of low molecular crystals.
\end{abstract}

KEY WORDS Thermal Conductivity/Cross-linking/Primary Bond / Van der Waals Bond/Specific Heat/Sound Velocity/Grueneisen's Number / Polymer /

From studies of thermal conductivity of linear amorphous polymers, it has become clear that this property is dependent on molecular weight ${ }^{1,2}$ and molecular orientation. ${ }^{3,4}$ These macroscopic parameters are essentially related to the energy transport through van der Waals and primary bonds. Eiermann and Hellwege, et al.,$^{3-5}$ pointed out that the thermal conductivity through the primary bonds is about ten times greater than that through the van der Waals bonds. The orientation effect, as a typical example, has been explained in terms of the "elementary" resistances of these bonds. By stretching the polymer chain, primary bonds are aligned in the direction of stretching and van der Waals bonds perpendicular to it, so that the thermal conductivity increases in the direction of stretching and decreases in the direction perpendicular to it.

When a polymer is cross-linked by chemical methods or irradiation, the effects of crosslinking are observed in some physical properties of the polymer such as the specific volume or the glass transition temperature. ${ }^{6}$ These effects have been discussed from the standpoint of the change in the molecular weight of segments between cross-links. However, it is also possible to discuss these effects on the basis of the change in the relative number of the van der Waals bonds (or primary ones), because crosslinking replaces some van der Waals bonds with covalent ones. Recently, the thermal conductivity of some cross-linked polymers has been investigated and found to increase with increasing degrees of cross-linking. ${ }^{7,8}$

In this paper we will discuss theoretically the effect of cross-linking on the thermal conductivity, using the concept presented by Eiermann and Hellwege, et al., in the interpretation of the thermal conductivity for linear amorphous polymers.

\section{THEORETICAL CONSIDERATIONS}

\section{Theoretical Equation for the Thermal Conduc- tivity for Cross-linked Polymers}

An equation for estimating the effect of crosslinking on the thermal conductivity of polymers can be derived, using the network model presented by Eiermann and Hellwege, et al.,${ }^{3-5}$ for the thermal conduction of linear amorphous polymers. On the macroscopic basis, it is reasonable to assume that one-third of polymer segments are oriented in one direction, while the remaining two-thirds are oriented in a direction perpendicular to the first segments (see 


\section{O. Yamamoto}

Figure 8 in ref 8 ). When a thermal gradient exists in the polymer, the over-all thermal resistance of the polymer is believed to result from the separate contributions, $R_{1}$ and $R_{2}$, of the polymer segments vertical and parallel to the direction of the thermal gradient. Then $R$, the over-all thermal resistance, is given by

$$
3 R=2 R_{1}+R_{2}
$$

As has been discussed in a previous paper, ${ }^{8}$ only $R_{1}$ will be altered by introducing cross-links into the polymer. However, in the direction of the thermal gradient one-half of the value of the cross-linking parameter $X=M_{\mathrm{g}} / M_{\mathrm{c}}$ should be accounted for, where $M_{\mathrm{g}}$ is the molecular weight of a chain unit and $M_{\mathrm{c}}$ is that of the chain segments between cross-links. The thermal resistances $R_{1}$ and $R_{2}$ are therefore described as follows

$$
\frac{1}{R_{1}}=\frac{X / 2}{R_{\mathrm{e}}}+\frac{1-X / 2}{R_{\mathrm{w}}} ; \quad R_{2}=R_{\mathrm{e}}
$$

$R_{\mathrm{W}}$ and $R_{\mathrm{e}}$ are the thermal resistances of the van der Waals and primary bonds. Inserting eq 2 in eq 1 , we get the over-all thermal resistance of the cross-linked polymer:

$$
3 R=\frac{(2+X / 2) R_{\mathrm{e}} R_{\mathrm{w}}+(1-X / 2) R_{\mathrm{e}}{ }^{2}}{(X / 2) R_{\mathrm{w}}+(1-X / 2) R_{\mathrm{e}}}
$$

Since the thermal resistance is reciprocal to the thermal conductivity, eq 3 can be written as follows:

$$
\lambda_{\mathrm{c}}=\frac{(X / 2) \lambda_{\mathrm{e}}+(1-X / 2) \lambda_{\mathrm{w}}}{(2+X / 2) \lambda_{\mathrm{e}}+(1-X / 2) \lambda_{\mathrm{w}}} \lambda_{\mathrm{e}}
$$

where $\lambda_{\mathrm{c}}$ is the thermal conductivity of the cross-linked polymer, $\lambda_{\mathrm{w}}$ and $\lambda_{\mathrm{e}}$ are the thermal conductivities through the van der Waals and primary bonds. If the parameter $X=0$, eq 4 becomes

$$
\frac{3}{\lambda_{0}}=\frac{1}{\lambda_{\mathrm{e}}}+\frac{2}{\lambda_{\mathrm{w}}}
$$

This is identical to the equation presented by Eiermann $^{5}$ for linear amorphous polymers. To compare the thermal conductivities of crosslinked and uncross-linked polymers, the following calculation was carried out. From eq 4 and 5 , the ratio of $\lambda_{c}$ to $\lambda_{0}$ is

$$
\begin{aligned}
\frac{\lambda_{\mathrm{c}}}{\lambda_{0}} & =\frac{(X / 2) P+(1-X / 2)}{(2+X / 2) P+(1-X / 2)}(2 P+1) \\
& =\frac{1+(P-1) X / 2}{1+\{(P-1) /(2 P+1)\} X / 2}
\end{aligned}
$$

The second term of the denominator is generally small, so that eq 6 can be expressed in the following form

$$
\begin{aligned}
\frac{\lambda_{\mathrm{c}}}{\lambda_{0}} & =\left\{1+(P-1) \frac{X}{2}\right\}\left\{1-\left(\frac{P-1}{2 P+1}\right) \frac{X}{2}\right\} \\
& =1-\frac{P^{2}-P}{2 P-1} X
\end{aligned}
$$

Since the value $P=\lambda_{\mathrm{e}} / \lambda_{\mathrm{w}}$ is approximately 10 , we get the simple equation:

$$
\frac{\lambda_{\mathrm{c}}}{\lambda_{0}}=1+4.3 X
$$

Thus, from eq 7 or eq 8 we can estimate theoretically the effect of cross-linking on thermal conductivity.

Theoretical Equation for Thermal Condutivity for Amorphous Copolymers

In this section we discuss the thermal conductivities, $\lambda_{\mathrm{e}}$ and $\lambda_{\mathrm{W}}$. In the following way data on the thermal conductivity of copolymers permits an estimation of the magnitude of $\lambda_{e}$ and $\lambda_{\mathrm{w}}$. In discussing the thermal conductivity of copolymers, it is necessary to define parameters $f_{\mathrm{a}}$ and $f_{\mathrm{b}}\left(f_{\mathrm{a}}=1-f_{\mathrm{b}}\right)$. They represent the fractions of monomer units $\mathrm{A}$ and $\mathrm{B}$ found in the copolymer chain:

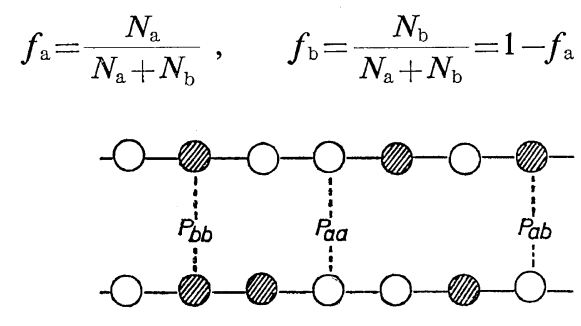

$$
\begin{aligned}
& \text { Number of Monomer Units } A \text { and } B \text { in the } \\
& \text { Copolymer Chain } \cdots-N_{a} \text { and } N_{b} \\
& f_{a}=\frac{N_{a}}{N_{a}+N_{b}}, f_{b}=\frac{N_{b}}{N_{a}+N_{b}}=1-f_{a} \\
& P_{a a}=f_{a}^{2}, P_{b b}=f_{b}^{2}, P_{a b}=2 f_{a} f_{b}
\end{aligned}
$$

Figure 1. Schematic representation of copolymer system. 
where $N_{\mathrm{a}}$ and $N_{\mathrm{b}}$ are the number of monomer units $\mathbf{A}$ and $\mathbf{B}$ in the copolymer chain. The probabilities $\boldsymbol{P}_{\mathrm{aa}}$ and $\boldsymbol{P}_{\mathrm{bb}}$ that the same monomer pair will be found in the two different polymer chains can be expressed as

$$
P_{\mathrm{aa}}=f_{\mathrm{a}}{ }^{2}=\left(\frac{N_{\mathrm{a}}}{N_{\mathrm{a}}+N_{\mathrm{b}}}\right)^{2} ; \quad P_{\mathrm{bb}}=f_{\mathrm{b}}{ }^{2}=\left(\frac{N_{\mathrm{b}}}{N_{\mathrm{a}}+N_{\mathrm{b}}}\right)^{2}
$$

The probability $\boldsymbol{P}_{\mathrm{ab}}$ for the different monomer pair is

$$
P_{\mathrm{ab}}=2 f_{\mathrm{a}} f_{\mathrm{b}}=\frac{2 N_{\mathrm{a}} N_{\mathrm{b}}}{\left(N_{\mathrm{a}}+N_{\mathrm{b}}\right)^{2}}
$$

As can be seen in Figure 1, the thermal resistance $R_{\text {w }}$ of the van der Waals bond, similar to that for the cross-linked polymer, is expressed by

$$
\frac{1}{R_{\mathrm{w}}}=\frac{\boldsymbol{P}_{\mathrm{aa}}}{\boldsymbol{R}_{\mathrm{aw}}}+\frac{\boldsymbol{P}_{\mathrm{bb}}}{\boldsymbol{R}_{\mathrm{bw}}}+\frac{\boldsymbol{P}_{\mathrm{ab}}}{\boldsymbol{R}_{\mathrm{abw}}}
$$

where $R_{\mathrm{aw}}, R_{\mathrm{bw}}$, and $R_{\mathrm{abw}}$ are the thermal resistances of the van der Waals bonds with the same monomer pair or a different one. Substituting eq 12 for $R_{1}$ in eq 1 and rewriting the equation of thermal conductivity, we obtain the following equation

$$
\lambda_{\mathrm{cop}}=\frac{3\left(\boldsymbol{P}_{\mathrm{aa}} \lambda_{\mathrm{aw}}+\boldsymbol{P}_{\mathrm{bb}} \lambda_{\mathrm{bw}}+\boldsymbol{P}_{\mathrm{ab}} \lambda_{\mathrm{abw}}\right)}{2+\boldsymbol{P}_{\mathrm{aa}}\left(\lambda_{\mathrm{aw}} / \lambda_{\mathrm{e}}\right)+\boldsymbol{P}_{\mathrm{bb}}\left(\lambda_{\mathrm{bw}} / \lambda_{\mathrm{e}}\right)+\boldsymbol{P}_{\mathrm{ab}}\left(\lambda_{\mathrm{abw}} / \lambda_{\mathrm{e}}\right)}
$$

If $\lambda_{\mathrm{abw}}$ can be expressed as an arithmetic mean value of $\lambda_{a w}$ and $\lambda_{b \mathbb{w}}$, eq 13 is simplified as

$$
\lambda_{\text {cop }}=\frac{3 f_{\mathrm{a}} \lambda_{\mathrm{aw}}+3 f_{\mathrm{b}} \lambda_{\mathrm{bw}}}{2+f_{\mathrm{a}}\left(\lambda_{\mathrm{aw}} / \lambda_{\mathrm{e}}\right)+f_{\mathrm{b}}\left(\lambda_{\mathrm{bw}} / \lambda_{\mathrm{e}}\right)}
$$

Indeed, it was found that eq 14 holds for the case of styrene-methyl methacrylate copolymer (ST-MMA).

Thermal Conductivity Obtained for the Heat Currents through the van der Waals and Primary Bonds

Recently, Knappe, et al., measured the thermal conductivity for some copolymers in the temperature range between -180 and $140^{\circ} \mathrm{C}$. In this study the calculation for estimating the values of $\lambda_{\mathrm{e}}$ and $\lambda_{\mathrm{w}}$ was made using the values of ST-MMA copolymer whose composition is known. In Figure 2 the solid line is the calculated curve obtained as the best fit of eq 13 to

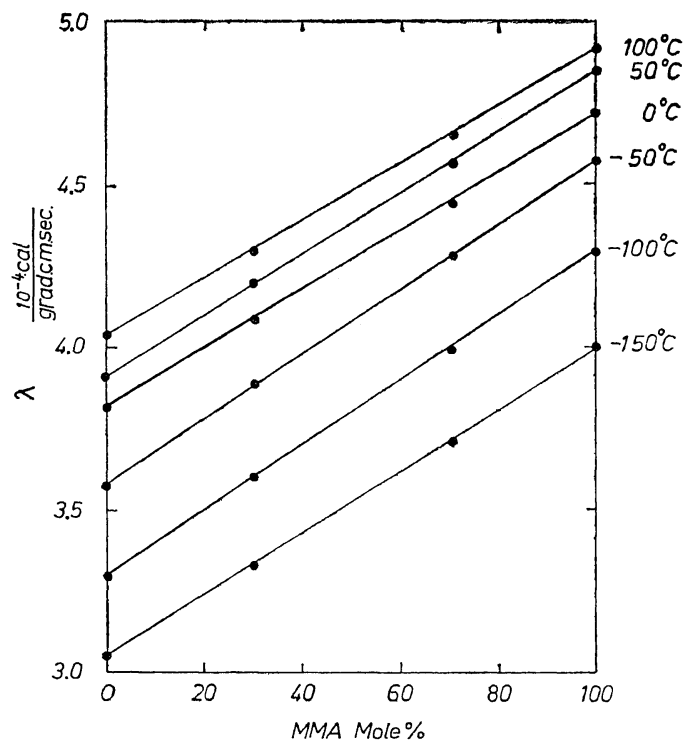

Figure 2. Thermal conductivity of styrene-methylmethacrylate copolymer: measured (data of Knappe, et al.); -, calculated.

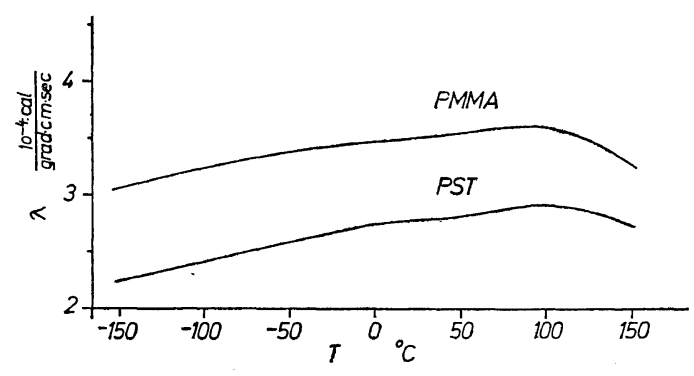

Figure 3. Temperature dependence of the thermal conductivity through the van der Waals bond.

the measured thermal conductivity. Figure 3 shows the temperature dependence of the calculated thermal conductivity for the van der Waals bond. In the temperature curve, a break was found at the glass transition temperature for both PMMA and PST. From this fact, the observed break for amorphous polymers at the glass transition temperature can be directly related to the state of the van der Waals forces. Figure 4 shows the temperature dependence of the thermal conductivity for the primary bond. For comparison, the thermal conductivity data of inorganic glasses are also plotted. ${ }^{10}$ The value at room temperature is in the order of $10^{-3}$ $\mathrm{cal} / \mathrm{grad} \mathrm{cm} \mathrm{sec}$, as well as those for the inor- 


\section{O. Yамамото}

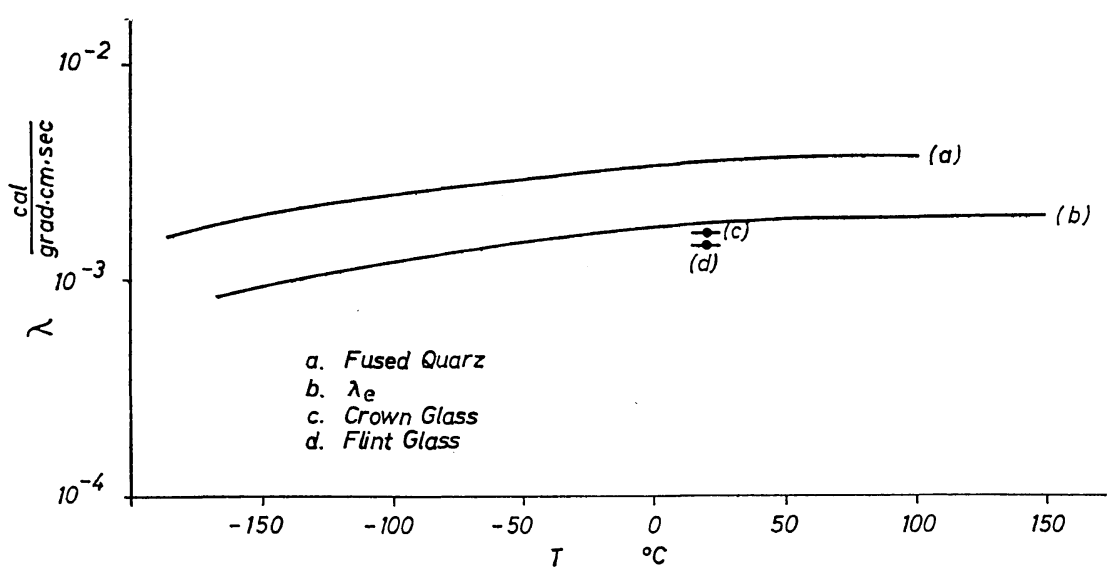

Figure 4. Temperature dependence of the thermal conductivity through the primary bond.

ganic glasses, which is about ten times greater than the thermal conductivity through the van der Waals bond. The thermal conductivity, unlike $\lambda_{\mathrm{w}}$, was found to increase with increasing temperature without any change at the glass transition temperature.

\section{Thermal Conduction through the van der Waals} Bond

According to the theory of thermal conductivity for low-molecular substances, ${ }^{10} \lambda$ is expressed as follows

$$
\lambda=\frac{1}{3} C_{v} \rho u l
$$

In this equation $C_{v}$ is the specific heat at constant volume, $\rho$ is the density, $u$ is the sound velocity and $l$ is the average-mean free path of phonons. There are two primary factors restricting the mean free path, ${ }^{11}$ namely, the interactions between the phonons themselves and the interactions between the phonons and defects, so that the mean free path depends on temperature and structure. For an amorphous substance at high temperature, the mean free path may be taken as a constant value independent of temperature. ${ }^{10}$ Consequently, the behavior of the thermal conductivity at high temperatures is characterized by the factors $C_{v}, u$, and $\rho$. Using this expression, the thermal conductivity through the van der Waals bond will be discussed as follows.

Specific Heat. The specific heat at constant volume may be calculated by using the thermo-
Table I. A values of poly(methyl methacrylate) and polystyrene

\begin{tabular}{ccc}
\hline Temp, ${ }^{\circ} \mathrm{C}$ & PMMA, g/cal & PST, g/cal \\
\hline 20 & $5.1 \times 10^{-4}$ & $6.9 \times 10^{-4}$ \\
106.7 & - & $7.3 \times 10^{-4}$ \\
\hline
\end{tabular}

dynamic relationship

$$
C_{p}-C_{v}=\frac{\alpha^{2} v T}{\beta}
$$

where $\alpha$ is the coefficient of thermal expansion, $\beta$ is the isothermal compressibility and $v$ is the specific volume. Specific heat at constant pressure $C_{p}$ is determined by experiment. If insufficient data for the evaluation of eq 16 are available, the following equation may be used for the estimation of $C_{v}$ from $C_{p}$ :

$$
C_{p}-C_{v}=C_{p}^{2} A T
$$

$A$ seems to be constant in the temperature range where no transition occurs. For PMMA and PST, we calculated $A$ values at two temperatures below and above the glass transition temperature (see Table I). The data necessary for the evaluation of $A$ values were taken from the literature. ${ }^{12-15}$ Figures 5 and 6 show the temperature dependence of the specific heat for PMMA and PST. The values $C_{v}$ were calculated with both eq 16 and eq 17. From Figures 5 and 6 , it is seen that $C_{v}$ contributed from lattice vibrations increases with increasing temperature without any increase 


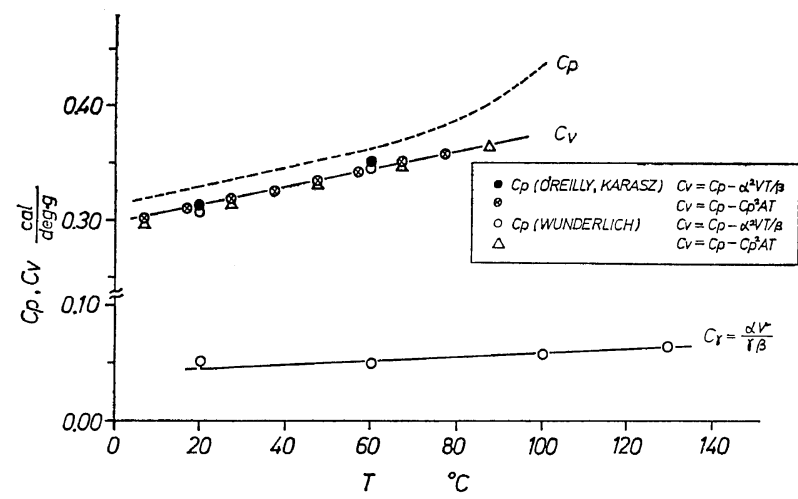

Figure 5. Specific heat of poly(methyl methacrylate).

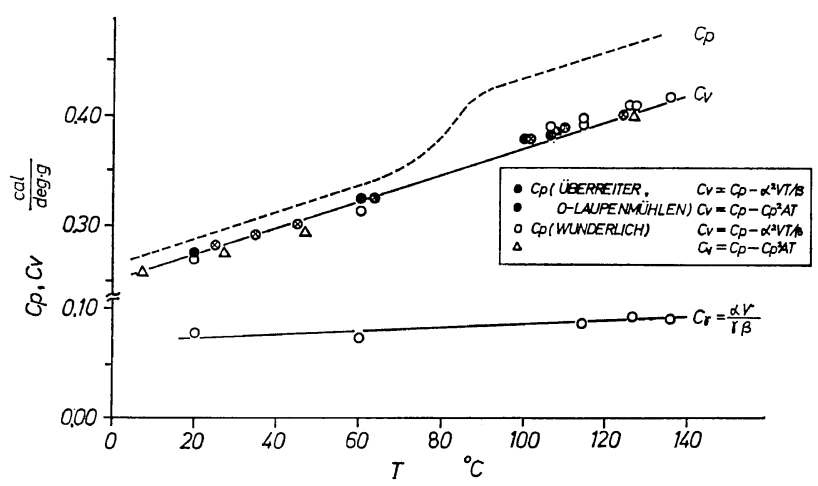

Figure 6. Specific heat of polystyrene.

at the glass transition temperature $T_{\mathrm{g}}$, while $C_{p}$ increases abruptly with hole formation at $T_{\mathrm{g}}$. For chain structure as found in linear polymers, the anisotropy of the forces must be considered. The forces between chains are weaker than the forces along the chain. Taking into account weak interactions between the chains, the specific heat $C_{v}$ can be divided into two elements, i.e., the contributions from the intra- and inter-chain vibrations. ${ }^{15,16}$ If we apply the specific heat at constant volume to the thermal conductivity through the van der Waals bond, we should have only the specific heat due to the interchain vibrations. ${ }^{16}$ It is known that the specific heat $C_{r}$ obtained from Grueneisen's equation corresponds to that due to the inter-chain vibrations. ${ }^{16}$ Grueneisen's equation is written as follows ${ }^{17}$

$$
\gamma_{G}=-\left(\frac{\partial \ln \Theta}{\partial \ln V}\right)_{T}=\frac{\alpha v}{C_{\gamma} \beta}
$$

where $\Theta$ is the Debye temperature and $\gamma_{G}$ is the Grueneisen number. According to Slater, ${ }^{18}$ the Grueneisen number can be expressed as

$$
\gamma_{\mathrm{G}}=\frac{a_{2}}{a_{1}{ }^{2}}-\frac{2}{3}
$$

In this expression $a_{1}$ and $a_{2}$ are the first and second compressibility coefficients in Bridgman's relation given by

$$
\frac{\Delta V}{V}=-a_{1} P+a_{2} P^{2}-\cdots
$$

where $\Delta V$ is the change in volume, $V$ is the original volume and $P$ is the pressure. The determinations of $a_{1}$ and $a_{2}$ for some typical polymers were made by using the compressibility data of Hellwege, et al. ${ }^{12}$ Figure 7 shows the Grueneisen number calculated with eq 19 for PST, PMMA, and high-pressure polyethylene. The values at room temperature are 3.8 for PST and PMMA. Recently, Wada, et al., ${ }^{16}$ reported 


\section{O. Үамамото}

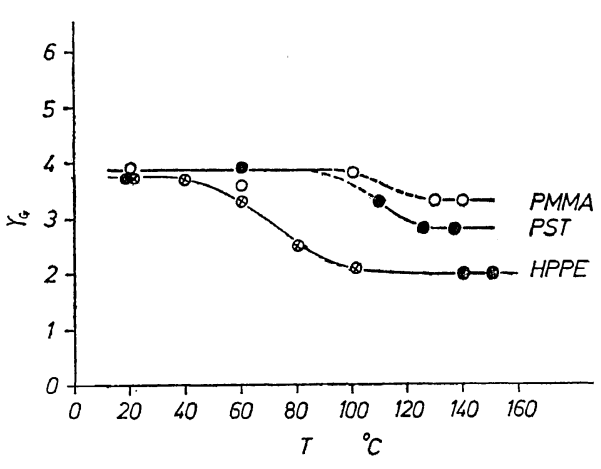

Figure 7. Grueneisen numbers for some polymers in the temperature range $20-150^{\circ} \mathrm{C}$.

the Grueneisen number determined from the pressure dependence of the sound velocity. They showed that the values at room temperature are about 4 for PST and PMMA. The values obtained here seem to give the lower limit. ${ }^{19}$ From Figure 7 it can be seen that the Grueneisen number is almost constant except for the change near the transition temperature. However, in any case, a remarkable decrease was found in the transition range. These results may be useful for a discussion of the temperature dependence of the sound velocity, which will be given later. From the $\gamma_{G}$ values, the specific heat $C_{\gamma}$ can be evaluated with eq 18. The results are also shown in Figures 5 and 6 . The calculated $C_{\gamma}$, as pointed out by Wada, et al., is quite small. The general behavior of $C_{r}$ is similar to $C_{v}$, but the temperature dependence of $C_{r}$ is smaller than that of $C_{v}$. When we discuss the specific heat of polymers, Tarasov's expression ${ }^{20}$ which consists of both one- and three-dimensional terms based on the anisotropic nature of the polymer chain, is applied to this property and is given by

$$
\frac{C_{v}}{R}=D_{1}\left(\frac{\Theta_{1}}{T}\right)-\frac{\Theta_{3}}{\Theta_{1}}\left[D_{1}\left(\frac{\Theta_{3}}{T}\right)-D_{3}\left(\frac{\Theta_{3}}{T}\right)\right]
$$

where $D_{n}$ is the $n$-dimensional Debye function and $\Theta$ 's are the Debye temperatures. At low temperatures, the inter-chain vibrations dominate and the specific heat of the polymer depends on the cube of the temperature, so that $C_{r}$ is closely approximated by the Debye function: ${ }^{11}$

$$
C_{r}=9 N K_{\mathrm{b}}\left(\frac{T}{\Theta}\right)^{3} \int_{0}^{\Theta / T} \frac{x^{4} e^{x}}{\left(e^{x}-1\right)^{2}} \mathrm{~d} x ; \quad\left(x=\frac{\hbar \omega}{K_{\mathrm{b}} T}\right)
$$

where $K_{\mathrm{b}}$ is the Boltzmann constant, $\hbar$ is the Planck constant divided by $2 \pi, N$ is the number of modes of vibrations and $\omega$ is the angular frequency.

Sound Velocity. From the Debye theory which predicts the cubic dependence of the specific heat on temperature at low temperatures, the Debye temperature is related to the sound velocity $^{11}$ as

$$
\Theta=\left(\frac{\hbar u}{K_{\mathrm{b}}}\right)\left(\frac{6 \pi^{2} N}{V}\right)^{1 / 3}
$$

Combining eq 18 and eq 23, we get the following relations

$$
u=\text { constant } V^{-\gamma+1 / 3}=K \rho^{\gamma-1 / 3}
$$

where $K$ is independent of temperature. The second equation is obtained by replacing $\rho=M / V$. Although $K$ is not known, it is possible to discuss the relative change of the sound velocity with temperature (or with the change of density). Figure 8 shows the temperature dependence of the relative sound velocity for PMMA and PST. $V_{0}$ is the sound velocity at $20^{\circ} \mathrm{C}$ taken as reference. The relative sound velocity decreases slightly with increasing temperature and an abrupt decrease is found in the glass transition range. The data for the ultrasonic sound velocity show similar change ${ }^{2,22}$ and the results

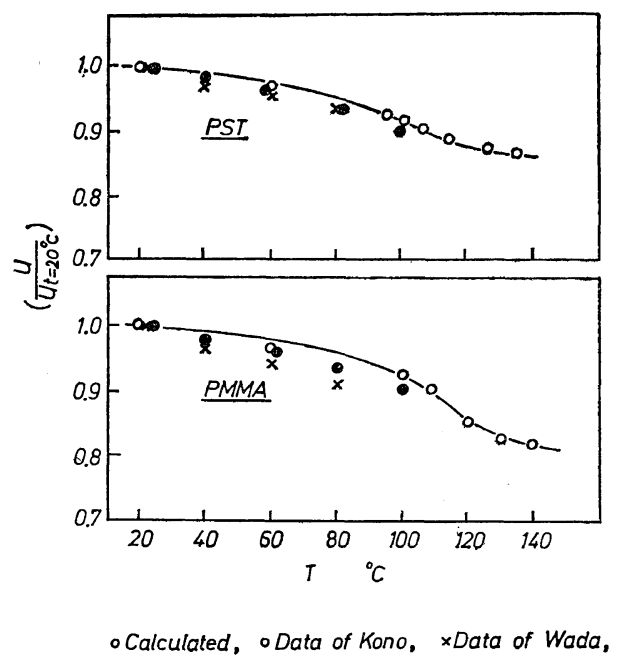

Figure 8. Temperature dependence of the relative sound velocity for polystyrene and poly(methyl methacrylate). 
obtained here seem to explain fairly well the measured sound velocity. As can be seen in Figure 3, the thermal conductivity through the van der Waals bond below $T_{\mathrm{g}}$ increases slightly with increasing temperature. A small increase of $\lambda_{\mathrm{w}}$ with temperature, based on the above discussion, can be attributed to that of the specific heat. On the other hand, in the glass transition range the sound velocity (or the density) decreases abruptly and hence causes a decrease in thermal conductivity. However, it may be expected from Figure 8 that the temperature dependence of $\lambda_{\mathrm{w}}$ at higher temperatures above $T_{\mathrm{g}}$ becomes smaller again. Sheldon and $\mathrm{Lane}^{23}$ pointed out this probability from the thermal conductivity data of poly(vinyl chloride).

\section{Thermal Conduction through the Primary Bond}

The behavior of $\lambda_{\mathrm{e}}$ is quite similar to that of inorganic glasses. The thermal conductivity of these substances has been discussed by Kittel. ${ }^{10}$ The results obtained by Kittel are also applied to the thermal conductivity through the primary bond. According to his discussion, the sound velocity was assumed to be independent of temperature, so that the increase of $\lambda_{e}$ with temperature can be attributed to the increase in specific heat. However, for polymers, the specific heat calculated from the one-dimensional Debye function may contribute to the thermal conductivity. This implies that a large amount of the difference in $C_{v}$ and $C_{r}$ may be incorporated into the thermal conduction through the primary bond.

\section{Some Remarks on the Discussion in the Previous} Section

When a factor affecting the thermal conductivity is introduced into a polymer, the resulting change arises only from the polymer segments vertical to the direction of the thermal gradient. We can then assume that the thermal conductivity of the polymer is approximately

$$
\lambda=K \lambda_{\mathrm{w}}
$$

where $K$ is independent of temperature and relates to the number of the van der Waals bonds. This assumption was first made by Eiermann ${ }^{5}$ to explain the thermal conductivity of polymers. For amorphous polymers at high temperatures, as discussed previously, we get the following equation from eq 15,24 , and 25

$$
\lambda=\text { constant } C_{\gamma} \rho^{\gamma+2 / 3}
$$

If the thermal conductivity accompanied with the change of the density is represented by $\lambda^{*}$, the equation of $\lambda^{*}$ may be given by

$$
\lambda^{*}=\text { constant } C_{r}(\rho+\Delta \rho)^{r+2 / 3}
$$

In this equation $\rho$ is expressed as $(\rho+\Delta \rho)$. It should be noted that eq 26 is valid only for the system where $C_{\gamma}$ is not affected by the introduced factor. Then the ratio of $\lambda^{*}$ to $\lambda$ can be calculated and we obtain as the equation relating to the change of the density:

$$
\begin{aligned}
\frac{\lambda^{*}}{\lambda} & =\left(\frac{\rho+\Delta \rho}{\rho}\right)^{\gamma+2 / 3}=\left(1+\frac{\Delta \rho}{\rho}\right)^{\gamma+2 / 3} \\
& =1-\left(\gamma+\frac{2}{3}\right) \frac{\Delta \rho}{\rho}=1+\varphi \frac{\Delta \rho}{\rho} ; \quad\left(\varphi=\gamma+\frac{2}{3}\right)
\end{aligned}
$$

By using a Grueneisen number below $T_{\mathrm{g}}$ for PST and PMMA, we obtain $\varphi=4.2-4.7$. The value of $\varphi$ seems to correspond to that of 4.3 in eq 8 .

Recently, Lohe ${ }^{5,24}$ investigated the pressure dependence on the thermal conductivity of polymers in the molten state. The results obtained by Lohe may support the above consideration. The equation describing the pressure dependence of the thermal conductivity can be derived from eq 26

$$
\frac{1}{\lambda}\left(\frac{\mathrm{d} \lambda}{\mathrm{d} P}\right)_{T}=\frac{1}{C_{\gamma}}\left(\frac{\mathrm{d} C_{\gamma}}{\mathrm{d} P}\right)_{T}+\varphi \frac{1}{\rho}\left(\frac{\mathrm{d} \rho}{\mathrm{d} P}\right)_{T}
$$

The first term at the right-hand side of eq 29 is not known. However, Bridgman ${ }^{25}$ found that $1 / C_{v}\left(\mathrm{~d} C_{v} / \mathrm{d} P\right)_{T}$ has an approximate value of $-4 \times 10^{-5} \mathrm{~cm}^{2} / \mathrm{kp}$ for organic liquids. If we apply this value to the first term of eq 29 , we get the experimentally determined $\varphi$ value. In this case the second term of eq 29 is calculated from the literature in the pressure range from 0 to $400 \mathrm{kp} / \mathrm{cm}^{2} .^{12}$ The theoretical $\varphi$ value can be obtained from the Grueneisen number above the transition temperature. The results for PMMA, PST, and high-pressure polyethylene are listed in Table II. It appears that the experimentally determined values agree with those calculated. 


\section{O. Үамамото}

Table II. Experimental and calculated $\varphi$ values near $140^{\circ} \mathrm{C}$ for poly(methyl methacrylate), polystyrene and high-pressure polyethylene

\begin{tabular}{lccccc}
\hline \multirow{2}{*}{ Material } & $\frac{1}{\lambda}\left(\frac{\mathrm{d} \lambda}{\mathrm{d} P}\right)_{T}$, & $\frac{1}{\lambda}\left(\frac{\mathrm{d} \lambda}{\mathrm{d} P}\right)_{T}-\frac{1}{C_{v}}\left(\frac{\mathrm{d} C_{v}}{\mathrm{~d} P}\right)_{T}$, & $\frac{1}{\rho}\left(\frac{\mathrm{d} \rho}{\mathrm{d} P}\right)_{T}$, & \multicolumn{2}{c}{$\varphi$} \\
& $10^{-5} \mathrm{~cm}^{2} / \mathrm{kp}$ & $10^{-5} \mathrm{~cm}^{2} / \mathrm{kp}$ & $10^{-5} \mathrm{~cm}^{2} / \mathrm{kp}$ & Expt1 & Calcd \\
\hline PMMA & 15 & 19 & 5.5 & 3.6 & $3.8-4.0$ \\
PST & 14 & 18 & 6.0 & 3.0 & $3.3-3.5$ \\
HPPE & 20 & 24 & 9.7 & 2.5 & $2.5-2.7$ \\
\hline
\end{tabular}

Theoretical Calculation of the Thermal Conductivity for Cross-linked Poly(methyl methacrylate) $(P M M A)$ and Polystyrene(PST)

Turning to the first and second sections of this paper, we estimate the effect of cross-linking on the thermal conductivity of polymers. By using eq 7 with the values of $\lambda_{e}$ and $\lambda_{w}$ obtained previously, we calculated the effect of cross-linking on the thermal conductivities of PMMA and PST in the range $X=0$ to $X=0.2$. The results can be seen in Figures 9 and 10 .

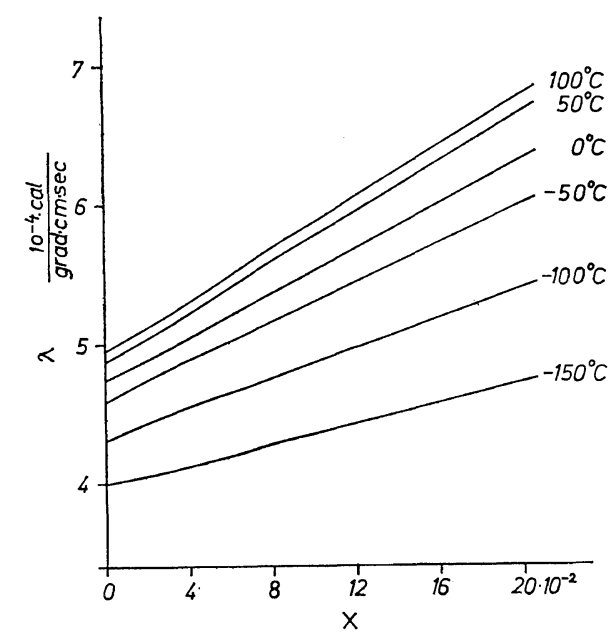

Figure 9. Theoretical calculation of the thermal conductivity for cross-linked poly(methyl methacrylate).

\section{CONCLUSIONS}

From the above discussion we arrive at the following conclusions. (1) Thermal conductivity increases with increasing degree of cross-linking. (2) The slope of the curves in Figures 9 and 10 becomes progressively greater as temperature increases. (3) The effect of cross-linking can be expected to be seen in the temperature curve

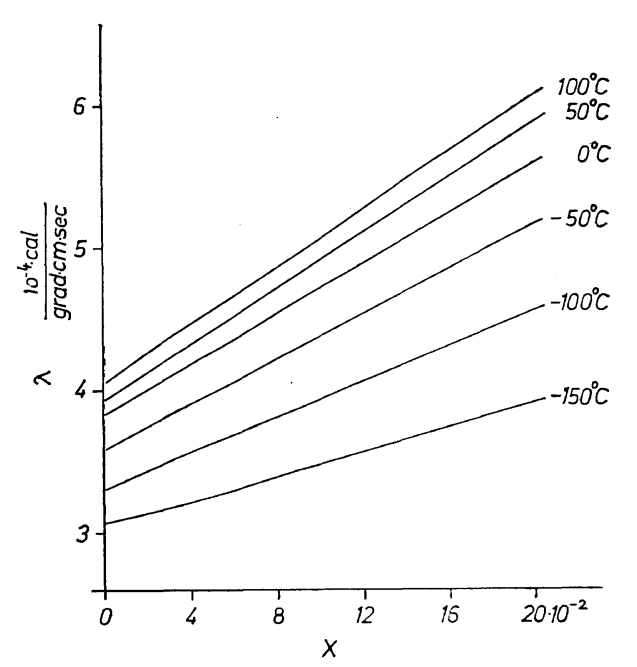

Figure 10. Theoretical calculation of the thermal conductivity for cross-linked polystyrene.

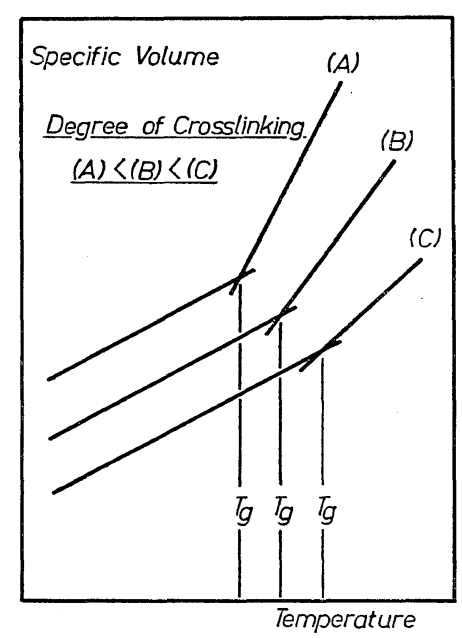

Figure 11. Temperature dependence of the specific volume for cross-linked polymer.

of thermal conductivity. Cross-linking decreases considerably the thermal expansion above $T_{\mathrm{g}}$, though the thermal expansion below $T_{\mathrm{g}}$ is not

Polymer J., Vol. 2, No. 4, 1971 
greatly affected by cross-linking ${ }^{6}$ (see Figure 11 ). This leads to the temperature coefficient above $T_{\mathrm{g}}$ becoming smaller with an increasing degree of cross-linking.

Acknowledgement. The author wishes to express his sincere gratitude to Professor H. Kambe for his valuable comments throughout this work.

\section{REFERENCES}

1. D. Hansen and C. C. Ho, J. Polym. Sci., Part A, 3, 659 (1965).

2. P. Lohe, Kolloid-Z. u. Z. Polymere, 204, 7 (1965).

3. K-H. Hellwege, J. Hennig, and W. Knappe, Kolloid-Z. u. Z. Polymere, 188, 121 (1963).

4. K. Eiermann, Kolloid-Z. u. Z. Polymere, 199, 125 (1964).

5. K. Eiermann, Kolloid-Z. u. Z. Polymere, 198, 5 (1964).

6. See, for example, L. E. Nielsen, Macromol. Sci.-Revs. Macromol. Chem., C3(1), 69 (1969).

7. J. N. Tomlinson, D. E. Kline, and J. A. Sauer, SPE Transactions, 5, 44 (1965).

8. W. Knappe and O. Yamamoto, Kolloid-Z. $u$. Z. Polymere, 204, 755 (1970).

9. W. Knappe, P. Lohe, and R. Wutschig, Angew. Makromol. Chem., 7, 181 (1969).
10. C. Kittel, Phys. Rev., 7S, 972 (1949).

11. C. Kittel, "Introduction to Solid State Physics", John Wiley and Sons, Inc., New York, N.Y., 1956.

12. K-H. Hellwege, W. Knappe, and P. Lehmann, Kolloid-Z. u. Z. Polymere, 183, 110 (1962).

13. K. Ueberreiter and S. Purucker, Kolloid-Z., 144, 120 (1955).

14. J. M. O'Reilly and F. E. Karasz, J. Polym. Sci., Part C, 14, 49 (1966).

15. B. Wunderlich and H. Baur, Adv. Polym. Sci., 7, 151-368 (1970).

16. Y. Wada, A. Itami, T. Nishi, and S. Nagai, J. Polym. Sci., Part A-2, 7, 201 (1969).

17. J. C. Slater, "Introduction to Chemical Physics", McGrow-Hill, New York, N.Y., 1939.

18. J. C. Slater, Phys. Rev., 57, 744 (1940).

19. R. E. Barker, J. Appl. Phys., 38, 4243 (1967).

20. V. V. Tarasov, J. Phys. Chem. (U.S.S.R), 24, 111 (1950).

21. Y. Wada, Oyobutsuri, 24, 159 (1955).

22. R. Kono, J. Phys. Soc. (Japan), 15, 718 (1960).

23. B. R. Sheldon and S. K. Lane, Polymer, 6, 77 (1965).

24. P. Lohe, Kolloid-Z. u. Z. Polymere, 203, 155 (1965); 205, 1 (1965); Dissertation, Darmstadt, 1965.

25. P. W. Bridgman, "The Physics of High Pressure', Bell Co., London, 1952. 